\title{
Statistical methods to correct for verification bias in diagnostic studies are inadequate when there are few false negatives: a simulation study
}

\author{
Angel M Cronin* and Andrew J Vickers
}

Address: Department of Epidemiology and Biostatistics and Department of Surgery, Memorial Sloan-Kettering Cancer Center, 1275 York Avenue, NY, NY 10021, USA

Email: Angel M Cronin* - serioa@mskcc.org; Andrew J Vickers - vickersa@mskcc.org

* Corresponding author

Published: II November 2008

BMC Medical Research Methodology 2008, 8:75 doi:10.1 186/147/-2288-8-75

This article is available from: http://www.biomedcentral.com/I47I-2288/8/75

(c) 2008 Cronin and Vickers; licensee BioMed Central Ltd.

This is an Open Access article distributed under the terms of the Creative Commons Attribution License (http://creativecommons.org/licenses/by/2.0), which permits unrestricted use, distribution, and reproduction in any medium, provided the original work is properly cited.
Received: 26 June 2008

Accepted: II November 2008

\begin{abstract}
Background: A common feature of diagnostic research is that results for a diagnostic gold standard are available primarily for patients who are positive for the test under investigation. Data from such studies are subject to what has been termed "verification bias". We evaluated statistical methods for verification bias correction when there are few false negatives.
\end{abstract}

Methods: A simulation study was conducted of a screening study subject to verification bias. We compared estimates of the area-under-the-curve (AUC) corrected for verification bias varying both the rate and mechanism of verification.

Results: In a single simulated data set, varying false negatives from 0 to 4 led to verification bias corrected AUCs ranging from 0.550 to 0.852 . Excess variation associated with low numbers of false negatives was confirmed in simulation studies and by analyses of published studies that incorporated verification bias correction. The $2.5^{\text {th }}-97.5^{\text {th }}$ centile range constituted as much as $60 \%$ of the possible range of AUCs for some simulations.

Conclusion: Screening programs are designed such that there are few false negatives. Standard statistical methods for verification bias correction are inadequate in this circumstance.

\section{Background}

A common feature of diagnostic research is that results for a diagnostic gold standard are available only for patients who are positive for the test under investigation. Prostatespecific antigen (PSA) testing is a typical example: we want to know the operating characteristics of the PSA test, but men are only recommended for biopsy (the gold standard assessment of prostate cancer) if their PSA is above a specified threshold, such as $4 \mathrm{ng} / \mathrm{ml}$. Accordingly we have little information on whether men with PSA less than $4 \mathrm{mg} / \mathrm{ml}$ do or do not have prostate cancer. True dis- ease state is therefore known for only a subset of participants, and because that subset is determined by the diagnostic test result, data are subject to what has been termed "verification bias"[1]. Verification bias is associated particularly with screening tests: screening a healthy population for a symptomless disease will, by definition, result in further diagnostic work up only for those with a positive screening test.

Begg and Greenes have proposed straightforward Bayesian methods to correct for verification bias $[1,2]$. Their 
method has been widely used [3-9] and requires only simple computations to estimate sensitivity and specificity for each threshold of the diagnostic test result, which can then be used to derive the area-under-the-receiver-operating-characteristics-curve (AUC). Alonzo and Pepe recently described another method for computing the AUC of a continuous screening test in the presence of verification bias. This method involves computing the sensitivity and specificity for each observed value of the screening test, but again is straightforward to implement[10]. Both of these methods rely on the assumption that data are missing at random[11], in other words, no other factor besides the diagnostic test result influenced verification status. Hunink et al have reported a method of correcting for verification bias when some participants receive the gold standard test based on variables other than the diagnostic test result, for example, if patients were sent to biopsy as a result of clinical findings. This method is similar to the method proposed by Begg and Greenes, but includes an additional modeling step [12].

One characteristic of many studies subject to verification bias, particularly those based on screening studies, is that only a very small number of participants with normal diagnostic test results will subsequently receive the gold standard assessment, that is, the number of false negatives is very low. It has previously been demonstrated that sensitivity cannot be accurately estimated in this scenario, even after correction for verification bias[13]. Here, we extend this argument to the area-under-the-receiver-operating-characteristic-curve. We also describe several previously published studies that involved verification bias correction, and examine whether their results might have been influenced by low false negative counts.

\section{Methods}

Take the case of study of screening for cancer, where the aim is to determine the relationship between results of the screening test and true disease status. Patients are screened using an imaging technology (the diagnostic test), and those with abnormal findings recommended for biopsy (the gold standard assessment). A hypothetical example from such a screening program is shown in Figure 1. A total of 500 patients are screened and 100 have abnormal findings. Since those with abnormal findings are strongly recommended to undergo biopsy, 75/100 decide to have a biopsy and 50/75 are confirmed to have disease present. Of the 400 patients with normal findings, 40 are nonetheless biopsied, and 5 are found to have disease.

To estimate the sensitivity and specificity of imaging for detecting cancer, the naive approach would be to use only data from biopsied patients. This results in a sensitivity of $91 \%(50 / 55)$ and a specificity of 58\% (35/60). However, it is obvious that patients with unfavorable characteristics (those likely to be both diagnostic and gold standard positive) are overrepresented and patients with favorable characteristics (those likely to be both diagnostic and gold standard negative) are underrepresented in the sample of biopsied patients. As a result, sensitivity is overestimated and specificity underestimated. This is a classic example of verification bias: imaging results are available for all 500 patients, but the gold standard available only for a subset, which is associated with the imaging result. Methods for verification bias correction, following Begg and Greenes [1] are given in the Appendix (see Additional file 1). Using these methods on our example data set, gives a corrected sensitivity of $57 \%(67 / 117)$ and specificity is $91 \%$ (350/ 383 ). Without verification bias correction, we would have concluded that imaging was highly sensitive but moderately specific when in fact the reverse is true.

Correction for verification bias becomes problematic if small cell counts are encountered. Table 1 gives some different scenarios for the biopsy results of patients with a normal imaging result in our cancer screening example. In the first row, the scenario shown in figure 1, 40 participants with a normal imaging result were biopsied, of which 5 were found with disease - that is, there were 5 false negatives - giving a corrected sensitivity of $57 \%$. In subsequent rows in table 1 , we vary the number of false negatives and find that small changes in the data lead to large differences in our estimates: a change from 2 to 1 false negatives, for example, increases sensitivity from $77 \%$ to $87 \%$. Clearly no robust statistical method should give such a different result given a change in status for a single patient in a 500 patient study.

A mathematical explanation for this observation is as follows. Consider the formula for the corrected sensitivity given in the appendix (see Additional file 1):

Corrected sensitivity $=\frac{\mathrm{v}_{11} \div\left[\left(\mathrm{v}_{11}+\mathrm{v}_{12}\right) \div \mathrm{n}_{1}\right]}{\mathrm{v}_{11} \div\left[\left(\mathrm{v}_{11}+\mathrm{v}_{12}\right) \div \mathrm{n}_{1}\right]+\mathrm{v}_{21} \div\left[\left(\mathrm{v}_{21}+\mathrm{v}_{22}\right) \div \mathrm{n}_{2}\right]}$

Here, $\mathrm{v}$ indicates patients with verified outcome (e.g. a biopsy result); $\mathrm{n}$ indicates all patients; the first and second subscripts refer to the test and gold standard results (e.g. imaging and biopsy) respectively; the subscript indicator 1 and 2 refer to test positive/disease and test negative/no disease. The problematic cell is the false negative cell, since participants are rarely verified if they have a strongly negative diagnostic test result; moreover, when these patients are verified, they are most likely to be disease free. It can be seen from (1) that as the false negative cell count $\left(\mathrm{v}_{21}\right)$ approaches zero, the second term in the denominator of (1) also approaches zero, resulting in a corrected sensitivity that approaches $100 \%$. 


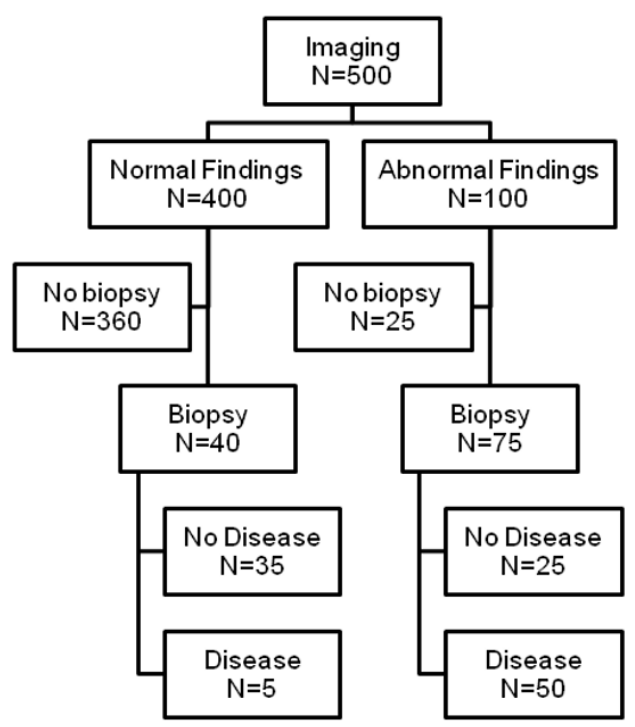

Summarized in contingency table form:

Before verification bias correction:

\begin{tabular}{l|ccccc} 
Imaging & All & \multicolumn{3}{|c}{ Biopsy Results } & Non-Biopsied \\
\cline { 3 - 5 } Result & Patients & Diseased & Nondiseased & Total & Patients \\
\hline Abnormal & 100 & 50 & 25 & 75 & 25 \\
Normal & 400 & 5 & 35 & 40 & 360 \\
\hline Total & 500 & 55 & 60 & 115 & 385
\end{tabular}

After verification bias correction:

\begin{tabular}{l|ccc}
\multirow{2}{*}{$\begin{array}{l}\text { Imaging } \\
\text { Result }\end{array}$} & \multicolumn{3}{|c}{ Biopsy Results } \\
\cline { 2 - 4 } Abnormal & Diseased & Nondiseased & Total \\
Normal & 50 & 33 & 100 \\
\hline Total & 117 & 350 & 400 \\
\hline
\end{tabular}

Figure I

Example of data subject to verification bias.

The sort of small differences in cell count which, as we have shown, can have a marked effect on estimates, are an inevitable consequence of sampling variability. In our principal example, 5 of the 40 patients with negative imaging results had positive biopsy. The $95 \%$ confidence interval for this proportion, $12.5 \%$, is $4 \%$ to $27 \%$ : accordingly it would not at all be unusual if, were we to repeat this experiment, we were to see only 2 of 40 patients with false negative results. In other words, in the imaging example we could have reported a sensitivity ranging from

Table I: Examples of data subject to verification bias and with a low number of false negatives

\begin{tabular}{ccccc}
\hline Example & \multicolumn{2}{c}{ Number with normal imaging result and biopsied } & Corrected Sensitivity* (\%) \\
\cline { 2 - 4 } & Diseased (False negatives) & Nondiseased (True negatives) & Total \\
\hline 1 & 5 & 35 & 40 & 57 \\
2 & 4 & 36 & 40 & 63 \\
3 & 3 & 37 & 40 & 77 \\
4 & 2 & 38 & 40 & 87 \\
6 & 1 & 39 & 40 & 100 \\
\hline
\end{tabular}

* Corrected for verification bias 
approximately $40 \%$ to $75 \%$ due to small chance differences in the number of false negatives, and there would be a very wide confidence interval around these estimates.

To investigate further the effects of low false negative counts on sensitivity, and in turn the AUC, we performed the following experiment:

A) We created a simulated data set with 5000 subjects. Both diagnostic and gold standard test results were known for all 5000 subjects, constituting a fully verified data set. Since the gold standard result was known for all subjects, we were able to fix the true AUC to 0.750. Data were simulated according to the specified probability models:

a. The gold standard test result follows a Bernoulli distribution with the mean equal to the incidence of disease, which was set to $10 \%$.

b. The diagnostic test result follows a log normal distribution where the $\log$ (test result) has a standard deviation of 1 and a mean of 0 and 1, respectively, for patients with negative and positive gold standard test results.

B) We introduced verification bias to the data in step A such that a certain proportion $v$ of participants were veri- fied, where $v$ was varied as an experimental parameter. The probability $p$ of verification for each subject increased with the diagnostic test result using the formula $\log [p /(1-$ $p)]=\alpha+0.5 d$, where $d$ was the decile of the diagnostic test result and the constant $\alpha$ adjusted to fix the overall probability of verification to $v$. This gives the probabilities shown in the top half of table 2 . We then applied a correction for verification bias, as shown in the appendix. Note that verification status depended solely on the diagnostic test result, therefore fulfilling the missing at random assumption required for this method. Since the diagnostic test in our simulation has a continuous distribution, the sensitivity and specificity was derived for multiple thresholds by dichotomizing the subjects into abnormal (above the threshold) and normal (below the threshold). A receiver operating characteristics curve was then constructed from these estimates [2] to calculate an AUC corrected for verification bias.

C) We repeated step B five times. Since we introduced verification bias in the same manner each time, we would expect no important differences in data structure between replications. Using the same argument, we would expect no important difference in verification bias corrected AUC unless standard methods were not appropriate for these data.

Table 2: Probability of verification used in the simulations for each decile of the diagnostic test result

\begin{tabular}{|c|c|c|c|}
\hline \multirow[t]{2}{*}{ Decile of diagnostic test result } & \multicolumn{3}{|c|}{ Probability of having the gold standard result (\%) } \\
\hline & $10 \%$ verified & $30 \%$ verified & $60 \%$ verified \\
\hline \multicolumn{4}{|c|}{ Probabilities of verification likely to be encountered in a screening study } \\
\hline I & $0.6 \%$ & $3.0 \%$ & $16.0 \%$ \\
\hline 2 & $0.9 \%$ & $4.9 \%$ & $23.9 \%$ \\
\hline 3 & $1.5 \%$ & $7.8 \%$ & $34.2 \%$ \\
\hline 4 & $2.5 \%$ & $12.2 \%$ & $46.1 \%$ \\
\hline 5 & $4.1 \%$ & $18.7 \%$ & $58.5 \%$ \\
\hline 6 & $6.5 \%$ & $27.5 \%$ & $69.9 \%$ \\
\hline 7 & $10.3 \%$ & $38.5 \%$ & $79.3 \%$ \\
\hline 8 & $15.9 \%$ & $50.7 \%$ & $86.3 \%$ \\
\hline 9 & $23.8 \%$ & $62.9 \%$ & $91.2 \%$ \\
\hline 10 & $34.0 \%$ & $73.7 \%$ & $94.5 \%$ \\
\hline \multicolumn{4}{|c|}{ Probabilities of verification were adjusted such that false negatives were more (less) likely to be present for $10 \%$ ( $60 \%$ ) verified } \\
\hline I & $6.4 \%$ & -- & $0.0 \%$ \\
\hline 2 & $7.0 \%$ & -- & $0.0 \%$ \\
\hline 3 & $7.7 \%$ & -- & $0.1 \%$ \\
\hline 4 & $8.5 \%$ & -- & $7.6 \%$ \\
\hline 5 & $9.3 \%$ & -- & $92.4 \%$ \\
\hline 6 & $10.2 \%$ & -- & $99.9 \%$ \\
\hline 7 & $11.1 \%$ & -- & $100.0 \%$ \\
\hline 8 & $12.1 \%$ & -- & $100.0 \%$ \\
\hline 9 & $13.2 \%$ & -- & $100.0 \%$ \\
\hline 10 & $14.4 \%$ & -- & $100.0 \%$ \\
\hline
\end{tabular}


D) We compared the true AUC from step A to the verification bias corrected AUC corresponding to each replication from step C.

The AUC was calculated using the trapezoid rule, where sensitivity and specificity were estimated (a) over 10 categories based on the deciles of the diagnostic test result and (b) for each unique value of the diagnostic test result using semiparametric efficient estimators - subsequently referred to as the Alonzo-Pepe method -, the latter of which has been shown to have minimal bias when the verification mechanism is known[10]. We specified that the simulated set have 5000 participants with $10 \%$ verification since these are common characteristics of large screening studies $[4,7,9]$.

We performed a simulation experiment where we repeated steps A and B 2000 times and report the mean of the true and verification bias corrected AUC, as well as the $2.5^{\text {th }}-97.5^{\text {th }}$ percentiles and coverage. Coverage was the proportion of $95 \%$ confidence intervals, constructed using bootstrap methods with 2000 replications, containing the true value of 0.750 . We performed this simulation experiment varying the proportion verified $(v=10,30$, and $60 \%$ ). Our intent in varying the proportion verified was to vary the frequency of the cell counts while keeping the relationship between the diagnostic test and outcome the same. For example, with all else being equal, one would be less likely to encounter small cell counts with $60 \%$ verified compared to $10 \%$ verified. To test whether small cell counts or overall verification rates drove our findings, we repeated our simulations using probabilities of verification as shown in the bottom half of table 2: in this case, the probability of small numbers of false negatives is higher in the scenario with a higher overall verification rate. For the simulations, the AUC was calculated by estimating sensitivity and specificity over 10 categories based on the deciles of the diagnostic test result; we did not calculate the AUC using the Alonzo-Pepe method as it gave similar results. All statistical analyses were conducted using Stata 9.2 (StataCorp, College Station, TX).

\section{Results \\ Simulation studies}

Data simulated under the setting of a screening study with 5000 participants are shown in Table 3. Defining "false negatives" as participants below the median diagnostic test level with a positive gold standard result, 99/2500 $(4.0 \%)$ were false negatives. Table 3 also shows five example runs of our simulation. After verification bias was randomly introduced the first time, only 50 ( $2 \%$ of 2500 ) participants below the median diagnostic test level had gold standard assessment of which 2 were false negatives. We introduced verification bias to the same simulated data set 4 more times. Comparing the replications, we observe no important differences in the proportion of participants below the median diagnostic test level who subsequently underwent gold standard assessment $(1.6 \%$ $2.6 \%)$. We also observe that the proportions of false negatives in each replication are consistent with chance when compared to the $4.0 \%$ of false negatives in the fully verified data set. Notably, the number of false negatives encountered ranged from $0-4$. AUC corrected for verification bias ranged from 0.550 to 0.852 . The variation in these results is very large: few estimates of AUC in the medical literature are less than 0.55 or greater than 0.85 . In other words, two replications of a screening study could produce results at opposite ends of the extremes of test characteristics. The estimates of AUC using the AlonzoPepe method showed similar gross variability, indicating that the variation in results is not explained by categorizing the continuous diagnostic test result in 10 groups. Similar results were observed when varying the true value

Table 3: Example of data generated under the setting of a screening study with $\mathbf{5 0 0 0}$ participants and the underlying incidence of disease being $10 \%$

\begin{tabular}{ccccc}
\hline Data Set & $\begin{array}{c}(1) \\
\text { Number with negative } \\
\text { diagnostic test and verified }\end{array}$ & $\begin{array}{c}(2) \\
\text { Number of false negatives }\end{array}$ & $\begin{array}{c}\text { Proportion of false } \\
\text { negatives }(2) /(1)\end{array}$ & AUC \\
Categorize in I0 bins & Alonzo-Pepe \\
\hline $\begin{array}{l}\text { Fully verified } \\
\text { With verification bias }\end{array}$ & 2500 & 99 & $4.0 \%$ & 0.750 \\
Replication I & $50(2.0 \%)$ & 2 & $4.0 \%$ & 0.750 \\
Replication 2 & $64(2.6 \%)$ & 4 & $6.3 \%$ & 0.852 \\
Replication 3 & $40(1.6 \%)$ & 2 & $5.0 \%$ & 0.550 \\
Replication 4 & $61(2.4 \%)$ & 0 & $0.0 \%$ & 0.812 \\
Replication 5 & $65(2.6 \%)$ & 3 & $4.6 \%$ & 0.857 \\
\end{tabular}

In the fully verified data set, definitive test results were known for all 5000 participants. In the replications with verification bias, only 500 (I0\%) participants underwent definitive testing. False negatives are defined as verified participants with a diagnostic test result less than the median of the diagnostic test results and with a positive gold standard result. The AUC of the fully verified data set was 0.750 ; all other estimates are with correction for verification bias. 
of the AUC, although less variation was present among highly predictive tests (for example, AUC of 0.9 , table 4).

The results of the simulation study are shown in table 5 . The number of false negatives is shown for a cut-off at the $2^{\text {nd }}$ decile. With $10 \%$ of participants verified, 0 false negatives were encountered in $83 \%$ of the replications; 1 false negative in $16 \%$, and $>1$ false negative in $2 \%$. The proportion of replications with $>1$ false negative increased as the percentage verified increased. With $30 \%$ of participants verified, $23 \%$ of replications had $>1$ false negative; this proportion rose to $92 \%$ with $60 \%$ of participants verified.

The verification bias corrected AUC had little bias: the mean over 2000 replications was generally close to the true AUC of 0.750. As expected, presence of verification bias increased the amount of variability associated with the AUC. With full verification, the $2.5^{\text {th }}-95^{\text {th }}$ percentiles of the AUC over 2000 replications were $0.728-0.774$; with $10 \%$ of participants verified, which is commonly observed in screening settings, this increased to 0.577 0.860 . The width of this interval, 0.28 , covers approximately $60 \%$ of all possible values of AUC. One way of illustrating these results is to note that a study with only 100 patients and a $10 \%$ event rate has a confidence interval for AUC of approximately 0.3: thus a study with 5000 patients that is subject to verification bias has equivalent statistical precision to one $98 \%$ smaller. Coverage was only $77 \%$ when $10 \%$ of patients were verified. As expected, both variability and coverage improved with a higher proportion verified: with $60 \%$ of participants verified, the $2.5^{\text {th }}-95^{\text {th }}$ percentiles of the AUC were 0.713 0.786 and coverage was $93 \%$.

Table 5 gives the results for the scenarios in the bottom half of table 2, where the number of false negatives is higher when the overall verification rate is lower. It is clear that the false negative count determines the value of verification bias correction: even if, overall, a reasonable number of patients are verified (60\%), our estimates have poor properties when there are few false negatives.

Table 4: Examples of estimates of verification bias corrected AUC when varying the true value of the AUC

\begin{tabular}{ccccc}
\hline & \multicolumn{5}{c}{ AUC } \\
\cline { 3 - 5 } Fully verified (True value) & 0.600 & 0.700 & 0.800 & 0.900 \\
\hline With verification bias correction & & & & \\
Replication I & 0.284 & 0.503 & 0.688 & 0.873 \\
Replication 2 & 0.754 & 0.838 & 0.742 & 0.894 \\
Replication 3 & 0.567 & 0.578 & 0.740 & 0.873 \\
Replication 4 & 0.689 & 0.779 & 0.883 & 0.920 \\
Replication 5 & 0.648 & 0.755 & 0.856 & 0.827
\end{tabular}

\section{Published example I - HPV, cervical cancer example}

In 2004, Dannecker et al examined the sensitivity of human papillomavirus (HPV) DNA on self-collected vaginal swabs for the diagnosis of cervical cancer [8]. The study included 435 participants in total, of whom 122 $(28 \%)$ underwent colposcopy, the gold standard assessment. The paper reported a sensitivity of $100 \%$ after verification bias correction, yet it is clear from examination of the paper that there were no false negatives. If instead there was 1 false negative (e.g. 1 participant with a negative diagnostic test result who was positive on colposcopy), the sensitivity after verification bias correction would be markedly reduced, from $100 \%$ to $70 \%$. Since there were zero false negatives, verification bias corrected confidence intervals cannot be derived (the authors did not provide confidence intervals, and did not mention the variability associated with the estimated sensitivity).

\section{Published example 2 - CAD, Single Photon Emission Computed Tomography}

In 2002, Miller et al examined the sensitivity of single photon emission computed tomography (SPECT) for the diagnosis of coronary artery disease (CAD) [7]. The study included 14,273 participants in total, of whom 1853 $(13 \%)$ underwent coronary angiography, the gold standard assessment. This paper reported a sensitivity of $65 \%$ (95\% confidence interval 63 to $68 \%$ ) after verification bias correction. There were 32 false negatives in the study. Small changes to the number of false negatives did not have a substantive difference in this example. For example, we estimate that changing the number of false negatives to 25 would result in a corrected sensitivity of $68 \%$, and to 40 would result in a corrected sensitivity of $62 \%$; these estimates are not importantly different from the reported sensitivity of $65 \%$, and are nearly within the reported 95\% confidence interval. From reviewing the statistical methods of the manuscript, we hypothesize that the reported confidence intervals are too narrow because the analysis included stepwise model selection, which was not replicated during the bootstrap procedure.

\section{Published example 3 - PSA, prostate cancer example}

In 2003, Punglia et al reported the AUC of a PSA test for prostate cancer diagnosis in a screening study conducted in the United States [4]. Of 6,691 men with PSA results, $705(11 \%)$ underwent biopsy of the prostate. The investigators performed a verification bias correction and reported the AUC of the PSA test for men aged $<60$ years to be 0.86 . From the presented tables and figures for men aged $<60$ years, we observe that the corrected sensitivity remained at $100 \%$ for PSA $<0.9 \mathrm{ng} / \mathrm{ml}$. This implies that there were no false negatives among men aged $<60$ years with PSA $<0.9 \mathrm{ng} / \mathrm{ml}$. Using simple assumptions (the median PSA among all 4556 men $<60$ screened being 0.9 $\mathrm{ng} / \mathrm{ml}$ and holding the specificity at this PSA level around 
Table 5: Simulation study with 2000 replications

\begin{tabular}{|c|c|c|c|c|c|c|c|}
\hline \multirow[t]{2}{*}{ Percentage Verified } & \multicolumn{4}{|c|}{ Proportion with $n$ false negatives } & \multicolumn{2}{|c|}{ AUC over 2000 replications } & \multirow[t]{2}{*}{ Coverage Probability over 2000 replications } \\
\hline & $n=0$ & $n=1$ & $n=2$ & $n>2$ & Mean & $2.5^{\text {th }}-97.5^{\text {th }}$ percentile & \\
\hline \multicolumn{8}{|c|}{ Probabilities of verification likely to be encountered in a screening study } \\
\hline True & $\mathrm{n} / \mathrm{a}$ & $\mathrm{n} / \mathrm{a}$ & $\mathrm{n} / \mathrm{a}$ & $\mathrm{n} / \mathrm{a}$ & 0.750 & $0.728,0.774$ & $95 \%$ \\
\hline $10 \%$ & $83 \%$ & $16 \%$ & $2 \%$ & $0 \%$ & 0.758 & $0.577,0.860$ & $77 \%$ \\
\hline $30 \%$ & $41 \%$ & $37 \%$ & $17 \%$ & $6 \%$ & 0.752 & $0.677,0.813$ & $89 \%$ \\
\hline $60 \%$ & $1 \%$ & $6 \%$ & $12 \%$ & $80 \%$ & 0.750 & $0.713,0.786$ & $93 \%$ \\
\hline \multicolumn{8}{|c|}{ Probabilities of verification were adjusted such that false negatives were more (less) likely to be present for $10 \%(60 \%)$ verified } \\
\hline $10 \%$ & $25 \%$ & $35 \%$ & $23 \%$ & $16 \%$ & 0.751 & $0.677,0.820$ & $93 \%$ \\
\hline $60 \%$ & $98 \%$ & $3 \%$ & $0 \%$ & $0 \%$ & 0.728 & $0.552,0.819$ & $67 \%$ \\
\hline
\end{tabular}

5000 participants are enrolled in a screening study with an underlying incidence of disease of $10 \%$. False negatives are defined as verified participants with a diagnostic test result less than the $20^{\text {th }}$ percentile of the diagnostic test results and with a positive gold standard result.

$55 \%$, as reported), and making a small change in false negatives, from 0 to 1 , the corrected sensitivity drops to $80 \%$. Had the AUC been calculated using $80 \%$ sensitivity instead of $100 \%$ sensitivity for PSA $<0.9 \mathrm{ng} / \mathrm{ml}$, then the corrected AUC would be 0.78 instead of 0.86 . Confidence intervals were not provided by the author for any sensitivity or AUC estimates.

\section{Discussion}

Verification bias is present in studies where only a subset of subjects receives the gold standard confirmation of disease status and where the likelihood of the gold standard confirmation depends on the diagnostic test result. These two conditions are often met in screening studies. When verification bias is not accounted for, reported sensitivity is inflated and specificity is understated. It is possible to obtain verification bias-corrected estimates of sensitivity and specificity if at least 1 subject with a negative screening test receives the definitive testing.

Several systematic reviews have investigated the prevalence of verification bias in diagnostic studies. In a review of pediatric studies published from 1987 to 1989, 40\% (15/42) were found to be verification bias[14]. Reviewing all diagnostic test studies published from 1978 to 1993, correction for verification bias was performed in 46\% (51/ 112) studies. Notably, the proportion of studies that corrected for verification bias significantly increased over time: 29\% from 1978-1981 and 62\% from 1990-1993. Finally, in a review of studies examining diagnostic tests for cancer published from 1990 to 2003, 40\% (10/25) at least mentioned verification bias as a potential source for bias[15].

It is important to recognize not only the need for but also the limitations of verification bias correction. In this paper, we illustrate that standard methods for verification bias correction are not adequate when there are few false negatives. This situation is commonly encountered in screening studies $[4,8]$. In these cases, verification bias correction would have led to dramatically different results given very small changes in the number of false negatives. Most skilled statisticians would be wary about applying statistical methods when there are low cell counts, and would therefore be concerned about the adequacy of verification bias correction in this circumstance. Yet we have shown that verification bias correction is often applied regardless of low numbers of false negatives. In the HPV example, the authors concluded that the HPV DNA test had excellent sensitivity, when in fact the reported sensitivity of $100 \%$ would have been $70 \%$ were a single patient to be reclassified. In the PSA screening example, the AUC could have been 0.78 instead of 0.86 if a single cancer were found among the estimated 2300 men with PSA < $0.9 \mathrm{ng} / \mathrm{ml}$. Although PSA is a screening test with a continuous distribution, it should be noted that verification bias-corrected estimates should not be obtained for PSA levels below which no man receives a biopsy. Indeed, using data from the Prostate Cancer Prevention Trial[16], a study in which men were biopsied irrespective of PSA level and thus not subject to verification bias, we might estimate well over 100 false negatives in the PSA $<0.9 \mathrm{ng} /$ ml group.

One possible solution to low false negative rates would be to provide confidence intervals, so that the width of the confidence interval can give insight into the certainty of the point estimate. Begg and Greenes provide formulas to calculate confidence intervals in the presence of verification bias, however, these formulas are based on asymptotic theory. In the case of few false negatives, the resulting confidence intervals will be insufficiently wide due to the 
uncertainty of the negative predictive value[13]. An obvious distribution-free method to calculate confidence intervals is by bootstrapping. However, since the false negative rate is underestimated in data subject to verification bias, we cannot be certain that resampling methods will sample from the true distribution of false negatives, in particular when the diagnostic test has a continuous distribution. For example, consider the case where the diagnostic test is continuous and there is only one false negative with a test result $\mathrm{t}=\mathrm{t}^{*}$ below a threshold value of the test $q$. Resampling methods will give a value $\mathrm{t}=\mathrm{t}^{*}$ for all diseased subjects below $q$, which is not the true distribution. As a result, bootstrap methods will provide overly narrow confidence intervals (table 5). In the HPV example, since the actual data did not contain any false negatives, no bootstrap sample would contain any false negatives; hence, the sensitivity would be overestimated in all bootstrap samples. Therefore, we do not believe that bootstrap methods to produce verification bias corrected confidence intervals are appropriate.

We have described the application of certain statistical methods in the presence of verification bias, and the problems with these methods when the analysis data set contains few false negatives. In other words, this manuscript focuses on the analytic phase of the study, and not the design phase. If it is known in advance that verification bias will be an issue, then a possible solution would be to design the study such that a random sample of test-negative subjects to undergo disease verification. The size of this sample would need to be sufficiently large to provide an adequate number of false negatives to estimate sensitivity, which may be difficult to determine in the original study design. In the CAD example, less than $2 \%$ of the test negative participants $(97 / 6745)$ were in the verified sample; a higher percentage of test negatives in the verified sample would be required in a smaller study. Although verification bias corrected estimates of diagnostic accuracy would be improved, this would come at a cost: subjects with no indication of disease would undergo unnecessary procedures, possibly resulting in complications and discomfort, not to mention the monetary cost of the additional procedures. The appropriateness of randomly selecting test-negative subjects to receive the gold standard assessment will be specific to the disease (for example, the incidence of disease and the invasiveness of the gold standard assessment. Since the focus of this manuscript is data analysis, and not study design, we have not made specific recommendations on this point.

For our simulations, we used data sets with 5000 subjects, a $10 \%$ incidence of disease and a verification rate as low as $10 \%$. These numbers were chosen to reflect the typical parameters of screening studies. For example, in one cervical cancer study, 364 of 4761 (8\%) women underwent biopsy [9]; the biopsy rate was 705 of 6691 (11\%) of men in a prostate cancer screening study; in a cardiovascular study 340/3679 (9\%) patients underwent angiography for disease status confirmation [5]. With respect to the true incidence of disease, this is naturally difficult to determine. As such, we conservatively chose a high incidence of $10 \%$ (note that, for example, the lifetime incidence of cervical cancer is less than $1 \%[17]$ ). It thus seems plausible that many screening studies would be prey to the problem of low false negative rates. The published examples (3.2 3.4 above) provide additional evidence on this point.

Previous mention has been made to the inadequacy of verification bias correction in the presence of low false negatives. In their critique of a novel imputation method, Hanley et al noted that sensitivity was $100 \%$ when there were no false negatives, even after verification bias correction. They cautioned against verification bias correction when one or more of the cells in the verified sample is zero [18]. Pepe has previously noted that verification bias corrected estimates of sensitivity are not robust to low numbers of false negatives[13]. We further demonstrate that standard methods to correct for verification bias, which have been widely used with zero false negatives, will produce unreliable estimates of sensitivity and AUC. Ultimately, verification bias is a missing data problem and we hypothesize that no correction method will be able to overcome a cell count of zero.

\section{Conclusion}

The characteristics of diagnostic tests have important clinical implications. From these results, clinicians decide whether or not to use a diagnostic test, and if so, how it should be used, such as what PSA cutoff to use to recommend patients for biopsy. Screening programs are designed such that few false negatives are encountered. When there are few false negatives, standard methods for verification bias correction are inadequate. If these methods are to be used, then at a minimum verification bias corrected confidence intervals should be provided for all estimates of diagnostic accuracy. Investigators should be cautioned when using such methods: there are no "free lunches" in statistics, and we should certainly be skeptical of methods that appear to get something from nothing.

\section{Competing interests}

The authors declare that they have no competing interests.

\section{Authors' contributions}

AMC and AJV conceived of the study, and participated in its design and coordination and helped to draft the manuscript. AMC performed the statistical analyses. All authors read and approved the final manuscript. 


\section{Additional material}

\section{Additional file 1}

Appendix verification bias

Click here for file

[http://www.biomedcentral.com/content/supplementary/1471-

2288-8-75-S1.doc]

\section{Acknowledgements}

Andrew Vickers' work was funded in part by a P50-CA92629 SPORE grant from the National Cancer Institute and by the Allbritton Fund. The funding body had no role in study design; in the collection, analysis or interpretation of data; in the writing of the manuscript; or in the decision to submit the manuscript for publication.

\section{References}

I. Begg CB, Greenes RA: Assessment of diagnostic tests when disease verification is subject to selection bias. Biometrics 1983, 39(I):207-2I5.

2. Gray R, Begg CB, Greenes RA: Construction of receiver operating characteristic curves when disease verification is subject to selection bias. Med Decis Making I984, 4(2): I5I-I64.

3. Merl T, Scholz M, Gerhardt P, Langer M, Laubenberger J, Weiss HD, Gehl HB, Wolf KJ, Ohnesorge I: Results of a prospective multicenter study for evaluation of the diagnostic quality of an open whole-body low-field MRI unit. A comparison with high-field MRI measured by the applicable gold standard. Eur J Radiol 1999, 30(I):43-53.

4. Punglia RS, D'Amico AV, Catalona W], Roehl KA, Kuntz KM: Effect of verification bias on screening for prostate cancer by measurement of prostate-specific antigen. N Engl J Med 2003, 349(4):335-342.

5. Roger VL, Pellikka PA, Bell MR, Chow CW, Bailey KR, Seward JB: Sex and test verification bias. Impact on the diagnostic value of exercise echocardiography. Circulation 1997, 95(2):405-4I0.

6. Lauer MS, Murthy SC, Blackstone EH, Okereke IC, Rice TW: [18F]Fluorodeoxyglucose uptake by positron emission tomography for diagnosis of suspected lung cancer: impact of verification bias. Arch Intern Med 2007, 167(2): I6I-165.

7. Miller TD, Hodge DO, Christian TF, Milavetz J], Bailey KR, Gibbons $\mathrm{RJ}$ : Effects of adjustment for referral bias on the sensitivity and specificity of single photon emission computed tomography for the diagnosis of coronary artery disease. Am J Med 2002, I I 2(4):290-297.

8. Dannecker C, Siebert U, Thaler C], Kiermeir D, Hepp H, Hillemanns $P$ : Primary cervical cancer screening by self-sampling of human papillomavirus DNA in internal medicine outpatient clinics. Ann Oncol 2004, I 5(6):863-869.

9. Schneider A, Hoyer H, Lotz B, Leistritza S, Kuhne-Heid R, Nindl I, Muller B, Haerting J, Durst M: Screening for high-grade cervical intra-epithelial neoplasia and cancer by testing for high-risk HPV, routine cytology or colposcopy. Int J Cancer 2000, 89(6):529-534.

10. Alonzo TA, Pepe MS: Assessing accuracy of a continuous screening test in the presence of verification bias. Applied Statistics 2005, 54:173-190.

II. Little RJ, Rubin DB: Statistical Analysis with Missing Data New York: Wiley; 1978.

12. Hunink MG, Richardson DK, Doubilet PM, Begg CB: Testing for fetal pulmonary maturity: ROC analysis involving covariates, verification bias, and combination testing. Med Decis Making 1990, I0(3):20I-2II.

13. Pepe MS: The Statistical Evaluation of Medical Tests for Classification and Prediction Oxford: Oxford University Press; 2003.

14. Bates AS, Margolis PA, Evans AT: Verification bias in pediatric studies evaluating diagnostic tests. J Pediatr 1993, 122(4):585-590.
15. Mallett S, Deeks J], Halligan S, Hopewell S, Cornelius V, Altman DG: Systematic reviews of diagnostic tests in cancer: review of methods and reporting. Bmj 2006, 333(7565):4I3.

16. Thompson IM, Ankerst DP, Chi C, Goodman PJ, Tangen CM, Lucia MS, Feng Z, Parnes HL, Coltman CA Jr: Assessing prostate cancer risk: results from the Prostate Cancer Prevention Trial. Natl Cancer Inst 2006, 98(8):529-534.

17. American Cancer Society. Cancer Facts \& Figures 2007. Atlanta: American Cancer Society. 2007.

18. Hanley JA, Dendukuri N, Begg CB: Multiple imputation for correcting verification bias by Ofer Harel and Xiao-Hua Zhou, Statistics in Medicine 2006; 25:3769-3786. Stat Med 2007 26(I5):. author reply 3047-3050.

\section{Pre-publication history}

The pre-publication history for this paper can be accessed here:

http://www.biomedcentral.com/1471-2288/8/75/prepub
Publish with Biomed Central and every scientist can read your work free of charge

"BioMed Central will be the most significant development for disseminating the results of biomedical research in our lifetime. "

Sir Paul Nurse, Cancer Research UK

Your research papers will be:

- available free of charge to the entire biomedical community

- peer reviewed and published immediately upon acceptance

- cited in PubMed and archived on PubMed Central

- yours - you keep the copyright 